\title{
Knowledge and attitudes of health care workers from intensive care units regarding nosocomial transmission of influenza: a study on the immediate pre-pandemic period
}

Fortaleza CR (1), Fortaleza CMCB (2)

(1) Course of Health Care Infections, Federal University of São Paulo, UNIFESP, São Paulo, São Paulo State, Brazil; (2) Department of Tropical Diseases, Botucatu Medical School, São Paulo State University (UNESP - Univ Estadual Paulista), Botucatu, São Paulo State, Brazil.

\begin{abstract}
The transmission of influenza in health care settings is a major threat to patients, especially those with severe diseases. The attitude of health care workers (HCWs) may influence the transmission of countless infections. The current study aimed to quantify knowledge and identify attitudes of HCWs involved in intensive care units (ICUs) regarding the risk of nosocomial influenza transmission. A questionnaire was applied through interviews to HCWs who worked in one of the five ICUs from a teaching hospital. Questions about influenza were deliberately dispersed among others that assessed several infectious agents. Fortytwo HCWs were interviewed: nine physicians, ten nurses and 23 nursing technicians or auxiliaries. Among the $42 \mathrm{HCWs}, 98 \%$ were aware of the potential transmission of influenza virus in the ICUs, but only $31 \%$ would indicate droplet precautions for patients with suspected infection. Moreover, only $31 \%$ of them had been vaccinated against influenza in the last campaign (2008). Nursing technicians or auxiliaries were more likely to have been vaccinated, both by univariate and multivariable analysis. When asked about absenteeism, only $10 \%$ of the study subjects stated that they would not go to work if they had an influenzalike illness. Those findings suggest that, in non-pandemic periods, influenza control in hospitals requires strategies that combine continuous education with changes in organizational culture.
\end{abstract}

Key words: influenza, health care-acquired infections, health care workers, intensive care units, vaccination.

\section{INTRODUCTION}

The transmission of influenza within health services is extensively documented in the medical literature (1). Outbreaks have been reported in hospitals and nursing homes, even before the 2009-2010 pandemic wave (2-5). Furthermore, it is estimated that at least $20 \%$ of health care workers (HCWs) are infected with influenza virus every year, during autumn and winter months (6). Unfortunately, most HCWs continue to work during their illness. This is particularly worrisome in intensive care units (ICUs), where patients with severe underlying conditions may acquire influenza from infected staff - a phenomenom that has great impact on mortality (7).
Although vaccination against influenza is advised for HCWs, most studies report poor adherence to this recommendation. Vaccine coverage for most health care settings is less than $40 \%$ (8-10). Improving those rates is a major challenge for infection control practitioners. But the control of influenza transmission within hospitals also requires correct identification and timely isolation of patients with upper respiratory symptoms (11).

This study was designed to assess the knowledge and attitudes of HCWs from ICUs regarding important measures that are currently indicated to prevent nosocomial influenza. 


\section{SUBJECTS AND METHODS}

\section{Setting}

The study was conducted in the teaching hospital of Botucatu Medical School, São Paulo State University, UNESP, which has 400 active beds and five ICUs. The hospital has an Infection Control Committee and a Center for Immunization, which runs yearly campaigns of vaccination against influenza.

\section{Study Design}

A cross-sectional survey was performed based on interviews with a sample of HCWs from hospital ICUs. All the interviews were performed between September 2008 and March 2009. Therefore, the study was conducted immediately before the emergence of the 2009-2010 pandemics (which occurred in April 2009).

\section{Sample Size}

The sample size was estimated based on data of influenza vaccine coverage provided by the Center for Immunization. We assumed a population of $200 \mathrm{HCWs}$, and vaccine coverage of $15 \%$ ( $10 \%$ precision). Other parameters were: power of $80 \%$, alpha error of $5 \%$ and design effect of 1.0. The estimated size was 40 subjects, which was extended for 42 in order to achieve a better representation of all professional categories in the ICUs. Estimates were performed using OpenEpi ${ }^{\infty}$ software (Emory University, USA) (12).

\section{Inclusion and Exclusion Criteria}

Nurses, nursing technicians/auxiliaries and medical doctors working in any of the hospital ICUs were included in the study if they agreed to participate and signed the informed consent form. Medical residents, trainees, students and professionals who did not work in ICUs on a regular basis were excluded.

\section{Interviews}

A questionnaire was applied to the study subjects through interviews. The subjects were aware that they were participating on a study about transmission of infectious agents, but they did not know that the study focused on influenza. The questionnaire mixed questions about influenza and other agents. This procedure was applied to avoid bias in the HCW answers, and was fully approved by the Research Ethics
Committee. The questionnaire was divided into four parts. In the first one, demographics and professional data were collected. The second assessed HCW knowledge and attitudes regarding isolation precautions. Answers were analyzed in terms of agreement with the current guidelines of the Centers for Disease Control and Prevention (CDC) (11). However, since there is a disagreement concerning influenza between recommendations from the CDC (contact plus droplet precautions) and from World Health Organization and Brazilian Ministry of Health (droplet precautions only), we assumed both answers as "correct" (13). The third part assessed adherence to vaccination campaigns. Data about HCW absenteeism were collected in the fourth part.

\section{Statistical Analysis}

Data were stored and analyzed in Epi Info ${ }^{\circledR}$ for windows v.3.5 (Centers for Disease Control and Prevention, USA). All data were submitted to descriptive analysis for estimation of frequencies and means. Analytic statistics was performed to identify predictors for two outcomes: likelihood that the HCW would indicate precautions for droplet transmission for patients with suspected or confirmed influenza; and likelihood that the professional adhered to the 2008 influenza vaccination campaign. For both analyses, data were first submitted to univariate tests: Student's t-test or Mann-Whitney $U$ test for numeric variables; chi-square or Fisher's exact test for dichotomous variables. In a second step, they were included in multivariable hierarchical models of logistic regression (14). Data were introduced in the models in the following order: demographic data; professional information; place of work; knowledge and attitudes.

\section{Ethical Issues}

This study was fully approved by the Research Ethics Committee of Botucatu Medical School, UNESP. Moreover, all subjects included in the study signed an informed consent form.

\section{RESULTS}

Nine physicians, ten nurses and 23 nursing technicians or auxiliaries participated in the study. Their place of work was distributed as follows: medical-surgical ICU, 37\%; emergency ICU, 
$15 \%$; coronary ICU, 24\%; pediatric ICU, $12 \%$ and neonatal ICU (high risk nursery), 12\%. Among study subjects, $86 \%$ were females. The median age was 29 (range 22 to 49 ). Other median values of interest were: years since graduation - eight (range 1 to 24); years working in the ICU - four (range 0 to 23); weekly hours spent in the ICU 40 (range 12 to 64 ).

Ninety-eight percent of subjects were aware of the risk of influenza transmission in the hospital setting. However, only $31 \%$ would indicate precautions for droplet transmission for a patient with suspected or confirmed influenza, and none would indicate precautions for contact transmission. In general, HCWs made fewer mistakes in indication of precautions for infectious diseases other than influenza (Figure 1). In univariate analysis, professionals with greater number of working hours in the ICU tended to indicate droplet precautions (Table 1 ), but that finding was not confirmed in the multivariable model.

With respect to immunization, only $31 \%$ of HCWs were vaccinated against influenza in 2008 , and $39 \%$ did not adhere to any vaccination campaigns over the past five years. Only one subject was vaccinated five times in the last five years. This contrasted with the excellent coverage of hepatitis B vaccine among study subjects (95\%).

In univariate analysis, nursing technicians/

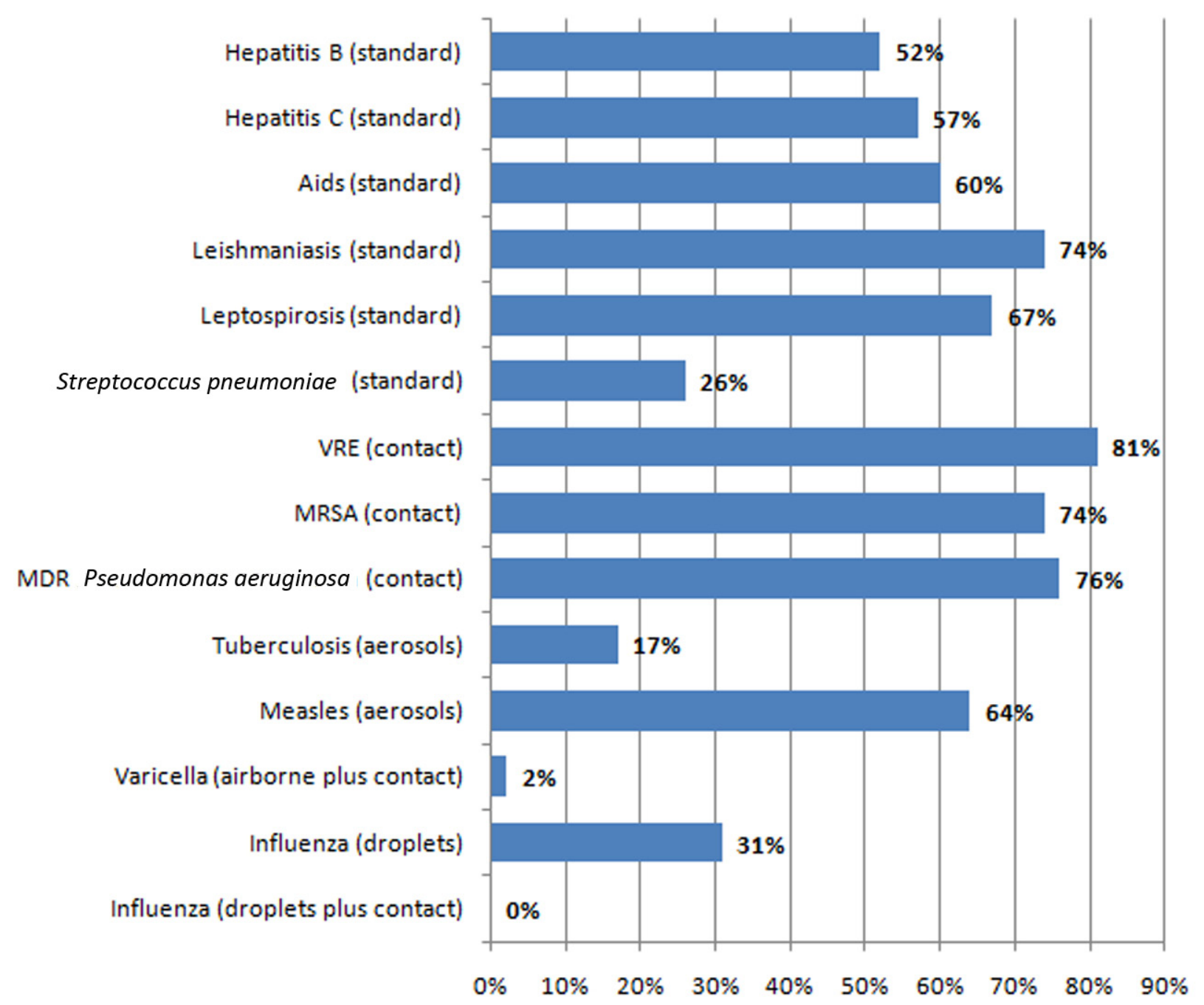

Figure 1. Proportion of subjects that would indicate isolation precautions according to CDC recommendations (in parenthesis) for different pathogens or diseases.

Note: for influenza, we considered both CDC recommendations (droplet plus contact) and World Health Organization guidelines (droplets). MDR: multidrug-resistant; MRSA: methicillin-resistant Staphylococcus aureus; VRE: vancomycin-resistant enterococci. 
Table 1. Factors predictive of indication of droplet precautions for influenza by study subjects

\begin{tabular}{|c|c|c|c|c|}
\hline Predictive factor & $\begin{array}{l}\text { HCWs who would } \\
\text { indicate droplet } \\
\text { precautions }(n=13)\end{array}$ & $\begin{array}{l}\text { HCWs who would not } \\
\text { indicate any isolation } \\
\text { precaution }(n=29)\end{array}$ & OR $(95 \% \mathrm{Cl})$ & $p$ \\
\hline \multicolumn{5}{|l|}{ Demographics } \\
\hline Male gender & $2(15 \%)$ & $4(14 \%)$ & $1.13(0.18-7.15)$ & 0.6 \\
\hline Age in years* & $29.5(22-49)$ & $29(23-41)$ & - & 0.9 \\
\hline \multicolumn{5}{|l|}{ Professional information } \\
\hline Medical doctor & $4(31 \%)$ & $5(17 \%)$ & $2.13(0.47-9.77)$ & 0.3 \\
\hline Nurse & $3(23 \%)$ & $7(24 \%)$ & $0.90(0.20-4.42)$ & 0.6 \\
\hline $\begin{array}{l}\text { Nursing technician/ } \\
\text { auxiliary }\end{array}$ & $6(46 \%)$ & $17(58 \%)$ & $0.61(0.16-2.26)$ & 0.3 \\
\hline Years since graduation* & $8(1-24)$ & $8(1-20)$ & - & 0.9 \\
\hline Years working in the ICU* & $4(0-23)$ & $4(0-20)$ & - & 0.6 \\
\hline $\begin{array}{c}\text { Weekly working hours in } \\
\text { the ICU* }\end{array}$ & $40(40-64)$ & $40(12-64)$ & - & $0.01^{* *}$ \\
\hline \multicolumn{5}{|l|}{ ICU } \\
\hline Medical-surgical & $4(30 \%)$ & $11(37 \%)$ & $0.72(0.18-2.04)$ & 0.5 \\
\hline Emergency & $0(0 \%)$ & $6(21 \%)$ & Undefined & 0.09 \\
\hline Coronary & $3(23 \%)$ & $7(24 \%)$ & $0.95(0.20-4.42)$ & 0.6 \\
\hline Pediatric & $2(15 \%)$ & $3(10 \%)$ & $\begin{array}{l}1.58(0.23- \\
10.78)\end{array}$ & 0.5 \\
\hline Neonatal & $3(23 \%)$ & $2(7 \%)$ & $\begin{array}{l}4.05(0.58- \\
27.92)\end{array}$ & 0.2 \\
\hline \multicolumn{5}{|l|}{ Knowledge and attitudes } \\
\hline $\begin{array}{l}\text { Correct indication of } \\
\text { isolation precautions for } \\
\text { other agents***, median } \\
\text { (range) }\end{array}$ & $6(2-10)$ & $7(3-10)$ & - & 0.2 \\
\hline $\begin{array}{l}\text { Vaccination against } \\
\text { influenza in } 2008\end{array}$ & $5(39 \%)$ & $8(28 \%)$ & $1.26(0.41-6.54)$ & 0.4 \\
\hline $\begin{array}{c}\text { Three or more vaccinations } \\
\text { against influenza in the } \\
\text { last five years }\end{array}$ & $4(31 \%)$ & $5(17 \%)$ & $2.13(0.47-9.77)$ & 0.3 \\
\hline $\begin{array}{l}\text { Cared for patients with } \\
\text { severe complications of } \\
\text { influenza }\end{array}$ & $2(15 \%)$ & $9(31 \%)$ & $0.40(0.07-2.21)$ & 0.3 \\
\hline $\begin{array}{l}\text { Would not work during } \\
\text { influenza-like illness }\end{array}$ & $1(8 \%)$ & $3(1 \%)$ & $0.72(0.07-7.68)$ & 0.6 \\
\hline
\end{tabular}

Note: data are presented in number of HCWs (with percentage in parenthesis), except for numeric variables. For those, which are marked with *, we present the median values and the range (minimum-maximum).

No variable was independently associated with the outcome in the multivariable logistic model.

${ }^{*}$ Numeric variable; ${ }^{* *}$ statistically significant; ${ }^{* *}$ number of indications coherent with CDC recommendations for 12 different infectious agents. 
auxiliaries and HCWs working in the coronary, 2008. In the multivariable model, only nursing emergency or pediatric ICUs were more likely technicians/auxiliaries were associated with to have been vaccinated against influenza in greater adherence (Table 2).

Table 2. Factors predictive of adherence of study subjects to the 2008 influenza vaccination campaign

\begin{tabular}{|c|c|c|c|c|}
\hline Predictive factor & $\begin{array}{l}\text { Adherence in } \\
2008 \\
(n=13)\end{array}$ & $\begin{array}{l}\text { Others } \\
(n=29)\end{array}$ & OR $(95 \% \mathrm{Cl})$ & $p$ \\
\hline \multicolumn{5}{|l|}{ Demographics } \\
\hline Male gender & $1(8 \%)$ & $5(17 \%)$ & $0.40(0.05-3.82)$ & 0.4 \\
\hline Age in years* & $29(22-40)$ & $29.5(23-49)$ & - & 0.6 \\
\hline \multicolumn{5}{|l|}{ Professional information } \\
\hline Medical doctor & $1(8 \%)$ & $8(27 \%)$ & $0.21(0.02-1.97)$ & 0.1 \\
\hline Nurse & $1(8 \%)$ & $9(31 \%)$ & $0.18(0.02-1.65)$ & 0.1 \\
\hline Nursing technician/auxiliary & $11(85 \%)$ & $12(41 \%)$ & $7.79(1.45-41.72)$ & $0.01 * * * * *$ \\
\hline Years since graduation* & $8(1-13)$ & $8(1-24)$ & - & 0.8 \\
\hline Years working in the ICU* & $3(1-9)$ & $5(0-23)$ & - & 0.1 \\
\hline $\begin{array}{l}\text { Weekly working hours in the } \\
\qquad \mathrm{ICU}^{*}\end{array}$ & $40(40-64)$ & $40(12-80)$ & - & 0.2 \\
\hline \multicolumn{5}{|l|}{ ICU } \\
\hline Medical-surgical & $2(15 \%)$ & $13(44 \%)$ & $0.22(0.02-1.19)$ & 0.06 \\
\hline Emergency & $4(31 \%)$ & $1(3.4 \% \%)$ & $12.4(1.23-126.18)$ & $0.03^{* *}$ \\
\hline Coronary & $0(0 \%)$ & $10(35 \%)$ & Undefined & $0.01^{* *}$ \\
\hline Pediatric & $4(31 \%)$ & $5(17 \%)$ & $12.40(1.23-126.18)$ & $0.03^{* *}$ \\
\hline \multicolumn{5}{|l|}{ Neonatal } \\
\hline \multicolumn{5}{|l|}{ Knowledge and attitudes } \\
\hline $\begin{array}{l}\text { Indicated droplet } \\
\text { precautions for } \\
\text { influenza**** }\end{array}$ & $5(39 \%)$ & $8(27 \%)$ & $1.64(0.41-6.54)$ & 0.4 \\
\hline $\begin{array}{l}\text { Cared for patients with } \\
\text { severe complications of } \\
\text { influenza }\end{array}$ & $4(31 \%)$ & 7 (24\%) & $1.39(0.33-5.97)$ & 0.5 \\
\hline $\begin{array}{l}\text { Knows people who had } \\
\text { negative experience with } \\
\text { vaccination }\end{array}$ & $12(92 \%)$ & $20(69 \%)$ & $5.30(0.66-48.08)$ & 0.1 \\
\hline $\begin{array}{l}\text { Knows people who had } \\
\text { positive experience with } \\
\text { vaccination }\end{array}$ & $9(69 \%)$ & $19(68 \%)$ & $1.18(0.29-4.83)$ & 0.5 \\
\hline $\begin{array}{l}\text { Would not work during } \\
\text { influenza-like-illness }\end{array}$ & 7 (54\%) & 17 (58\%) & $0.82(0.22-3.07)$ & 0.5 \\
\hline
\end{tabular}

Note: data are presented in number of HCWs (with percentage in parenthesis), except for numeric variables. For those, which are marked with *, we present the median values and the range (minimum-maximum).

*Numeric variable; ${ }^{* *}$ statistically significant; ${ }^{* * *}$ the category of nursing technician or auxiliary was an independent predictor of adherence to the 2008 campaign in the multivariable model $(\mathrm{OR}=7.79 ; 95 \% \mathrm{Cl}=1.36-41.7 ; \mathrm{p}=0.02){ }^{* * * *}$ see Figure 1 and Table 1 . 


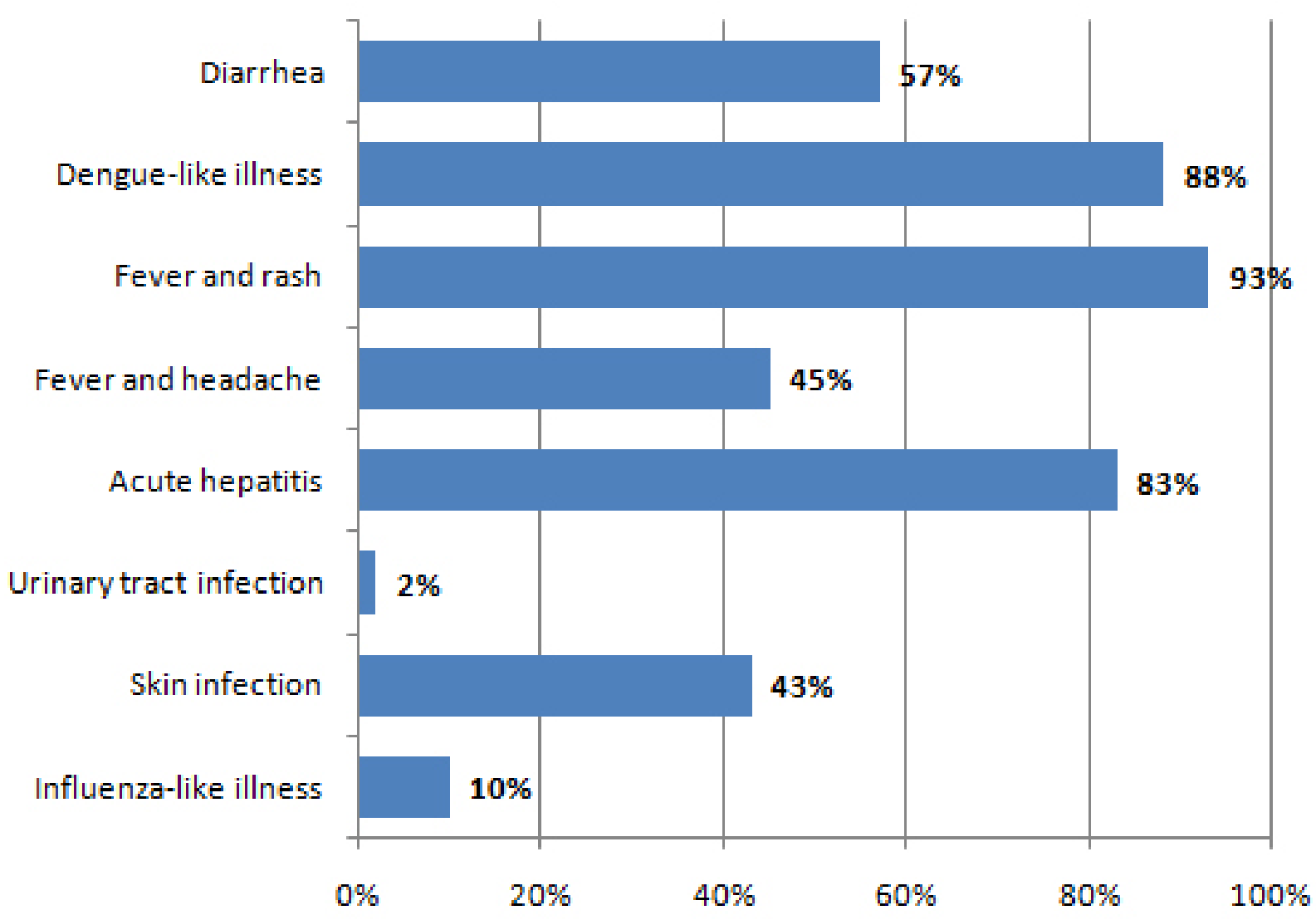

Figure 2. Proportion of study subjects who would not go to work during a presumed infectious disease.

In a stimulated question about actions that could improve adherence to influenza vaccination, all subjects mentioned the availability of vaccines in their working place. Other frequent answers were: improvement in visual marketing of the campaign, $81 \%$; more information from the Immunization Office, 78\%; beginning HCW vaccination simultaneously with the campaign for elderly, $62 \%$.

Only $10 \%$ of study subjects stated that they would not go to work if they had an influenzalike illness. Contrasted with attitudes in eight other situations of possible infectious diseases, this result was superior only to urinary tract infection (Figure 2).

In stimulated question, all the HCWs who would work during an influenza-like illness thought that absenteeism would undermine the work of the ICU team. Other answers were: "colleagues would not approve my absence", 96\%; "flu does not affect my ability to work", 83\%. Among the four HCWs who would not work, the only reason cited was "flu affects my ability to work".

\section{DISCUSSION}

The risk posed by influenza in the ICU setting is unquestionable. It is somewhat surprising that HCWs have historically overlooked this risk. Interestingly, in our study, almost all HCWs were aware of the potential dissemination of influenza virus within hospitals. Unfortunately, this finding is not corroborated by other results. Most HCWs would not indicate isolation precautions of any kind for a patient with suspected influenza. Only a few were vaccinated against influenza in 2008 and most would work normally during an influenza-like illness. These findings demonstrate that this is a matter of emphasis, not of ignorance. In general, HCWs tend to consider influenza a "mild" disease which is not worth infection control efforts. This is a cultural phenomenon, observed by several authors from different parts of the world $(1,2,3)$. Most findings from our study are coherent with this tendency. We shall discuss them in more detail bellow.

Only one third of HCWs interviewed for our study would indicate precautions against 
droplet transmission for patients with suspected or confirmed influenza. Worse, nobody would indicate precautions against droplets and contact transmission, as recommended by CDC. Since it was not possible to analyze the adherence do CDC recommendation, we attempted to identify predictors for indication of "droplet" precautions. No independent predictor was found in multivariable analysis.

Proper indication of isolation precautions is a field in which there are few studies. An interesting analysis was carried out by Sax et al. (15), regarding indications of isolation precautions for several infectious agents. The authors found greater knowledge of proper indications among people who received specific training, but also among younger HCWs. In our study, however, most HCWs would not isolate influenza patients properly, even though they were generally young, with ages ranging from 22 to 49 .

Adherence of HCWs to influenza vaccination is a matter of intense debate. Vaccine coverage was $31 \%$ among HCWs enrolled in our study. Even these subjects reported irregular adherence through the years. Indeed, all but one HCW had missed one or more vaccine campaigns in the last five years. These findings corroborate results from other authors $(8-10,16)$.

An interesting systematic review of articles addressing adherence to influenza vaccination was carried out by Hollmeyer et al. (17). The review extensively documented the poor adherence. Also, it analyzed reasons for "adherence" or "non-adherence" identified among subjects of 21 studies. The most cited reason for nonreceipt of influenza vaccine were "fear of adverse reaction", closely followed by "lack of concern". On the other hand, the most frequent reason for adhering to influenza vaccination campaigns was "self protection". "Protection of patients" ranked second, but with considerable distance. The authors conclude that, when HCWs get immunized against influenza, they do so for their own benefit - not for concern with patients' safety.

The adherence to influenza vaccination varies among professional categories. A study by Loulergue et al. (18) found greater adherence among medical doctors and HCWs caring for pediatric patients. On the other hand, a survey carried out in Bauru State Hospital, a facility affiliated to UNESP, found that medical doctors were less likely to have been vaccinated in the last campaign, and to have adhered to at least three campaigns in the last five years (19). Those findings are similar to ours. In our study, nursing technicians or auxiliaries were independently associated with greater adherence. Our results suggest that adherence is not related to knowledge or experience, but rather with the likelihood of following infection control recommendations.

Besides documenting the magnitude and predictors of non-adherence to influenza vaccine, we also attempted to identify HCW views about measures that could improve its coverage. There was a consensual opinion that providing access to the vaccine in the working place (the ICU) would be an effective way for ensuring adherence. The study subjects also mentioned the need of improving visual campaigns and information about the importance of the vaccine. Also, they pointed out to an interesting issue. The vaccination campaign against influenza for HCWs is usually initiated only after the annual vaccination for the elderly is finished. This gives the impression that they are receiving "surplus doses", hence they are not a priority category for immunization. It is noteworthy that $62 \%$ of respondents believed that the simultaneous onset of the two campaigns (HCWs and the elderly) would increase the adherence.

Influenza has been blamed for absenteeism in industries and health services (20). In the latter, the absenteeism is desirable, since HCWs working during influenza may infect the patients. According to our findings, most HCWs would not miss work because of influenza-like illness. Again, this fact demonstrates a mistaken assessment of the potential impact of influenza on the health of patients. Given the fact that that "influenza does not affect the ability to work", most professionals believed that "colleagues would not approve the absence".

Taken together, our findings suggest that in non-pandemic periods, influenza is considered a banal disease, with minor consequences to patients. Of course, the great number of admissions for severe pandemic influenza in 2009 changed the whole scene. But we firmly believe that our results are still valid. Now that the World Health Organization declared that the pandemic is over, it is expected that HCWs will once again neglect the influenza threat to critical patients just as they did in the pre-pandemic years. In order 
to improve attitudes towards influenza control, hospitals must provide continuous education for their HCWs and perform changes in institutional culture regarding vaccination and absenteeism. This is a major challenge for administrators and infection control teams - a challenge that saves lives.

\section{COPYRIGHT}

(c) CEVAP 2011

\section{SUBMISSION STATUS}

Received: November 16, 2010.

Accepted: March 1, 2011.

Abstract published online: March 10, 2011.

Full paper published online: May 31, 2011.

\section{CONFLICTS OF INTEREST}

There is no conflict.

\section{ETHICS COMMITTEE APPROVAL}

The present study was approved by the Research Ethics Committee of Botucatu Medical School, UNESP (protocol number 2901), and all subjects included signed an informed consent form.

\section{CORRESPONDENCE TO}

CRISTIANE RAVAGNANI FORTALEZA, Rua Professor Milton Guimarães, 311, Botucatu, SP, 18608-025, Brazil. Phone: +55 1438826668 . Email: cris.influenza@gmail.com.

\section{REFERENCES}

1. Voirin N, Barret B, Metzger MH, Vanhems P. Hospitalacquired influenza: a synthesis using the outbreak reports and intervention studies of nosocomial infection (ORION) statement. J Hosp Infect. 2009;71(1):1-14.

2. Salgado CD, Farr BM ,Hall KK, Hayden FG. Influenza in the acute hospital setting. Lancet Infect Dis. 2002;2(3):145-55.

3. Maltezou HC. Nosocomial influenza: new concepts and practice. Curr Opin Infect Dis. 2008;21(4):337-43.

4. Gomolin IH, Kathpalia RK. Influenza. How to prevent and control nursing home outbreaks. Geriatrics. 2002; 57(1):28-34.

5. Utsumi M, Makimoto K, Quroshi N, Ashida N. Types of infectious outbreaks and their impact in elderly care facilities: a review of the literature. Age Ageing. 2010;39(3):299-305.

6. Elder AG, O'Donnell B, McCruden EAB, Symington IS, Carman WF. Incidence and recall of influenza in a cohort of Glasgow healthcare workers during the 19934 epidemic: results of serum testing and questionnaire. British Med J. 1996;313(7067):1241-2.
7. Oliveira EC, Lee B, Colice GL. Influenza in the intensive care unit. J Intensive Care Med. 2003;18(2):80-91.

8. Talbot TR, Bradley SE, Cosgrove SE, Ruef C, Siegel JD, Weber DJ. Influenza vaccination of healthcare workers and vaccine allocation for healthcare workers during vaccine shortages. Infect Control Hosp Epidemiol. 2005;26(11):882-90.

9. Heimberger T, Chang HG, Shaikh M, Crotty L, Morse D, Birkhead G. Knowledge and attitudes of healthcare workers about influenza: why are they not getting vaccinated? Infect Control Hosp Epidemiol. 1995;16(7):412-5.

10. Lugo NR. Will carrots or sticks raise influenza immunization rates of health care personnel? Am J Infect Control. 2007; 35(1):1-6.

11. Siegel JD, Rhinehart E, Jackson M, Chiarello L. Health Care Infection Control Practices Advisory Committee. 2007 Guideline for Isolation Precautions: Preventing Transmission of Infectious Agents in Health Care Settings. Am J Infect Control. 2007; 35(Suppl 2): S65164.

12. Dean AG, Sullivan KM, Soe MM. OpenEpi: Open Source Epidemiologic Statistics for Public Health, Version 2.3. www.OpenEpi.com, updated 2009/20/05, accessed 2010/10/21.

13. Brasil. Ministério da Saúde. Protocolo de manejo clínico e vigilância epidemiológica da influenza. [Access 2010 August 20] Available from: http://portal. saude.gov.br/portal/arquivos/pdf/protocolo_de_ manejo_clinico_05_08_2009.pdf.

14. Greenland S. Bayesian perspectives for epidemiological research. II. Regression analysis. Int J Epidemiol. 2007;36(1):195-202.

15. Sax H, Perneger T, Hugonnet S, Herrault P, Chraït $\mathrm{MN}$, Pittet D. Knowledge of standard and isolation precautions in a large teaching hospital. Infect Control Hosp Epidemiol. 2005;26(3):298-304.

16. Takayanagi IJ, Cardoso MR, Costa SF, Araya ME, Machado CM. Attitudes of health care workers to influenza vaccination: why are they not vaccinated? Am J Infect Control. 2007;35(1):56-61.

17. Hollmeyer HG, Hayden F, Poland G, Buchholz U. Influenza vaccination of healthcare workers in hospitals - a review of studies on attitudes and predictors. Vaccine. 2009;27(30):3935-44.

18. Loulergue P, Moulin F, Vidal-Trecan G, Demontpion C, Menager C, Gorodetsky $M$ et al. Knowledge attitudes and vaccination coverage of healthcare workers regarding occupational vaccinations. Vaccine. 2009;27(31):4240-3.

19. Cavalcante RS, Jorge AM, Fortaleza CM. Predictors of adherence to influenza vaccination for healthcare workers from a teaching hospital: a study in the prepandemic era. Rev Soc Bras Med Trop. 2010;43(6):614-4

20. O'Reilly FW, Stevens AB. Sickness absence due to influenza. Occup Med. 2002;52(5):265-9. 